\title{
Philosophiques
}

\section{Le pouvoir de l'idéal et l'idéal du pouvoir}

\section{Joseph Pestieau}

Volume 8, numéro 2, octobre 1981

URI : https://id.erudit.org/iderudit/203169ar

DOI : https://doi.org/10.7202/203169ar

Aller au sommaire du numéro

Éditeur(s)

Société de philosophie du Québec

ISSN

0316-2923 (imprimé)

1492-1391 (numérique)

Découvrir la revue

Citer cet article

Pestieau, J. (1981). Le pouvoir de l'idéal et l'idéal du pouvoir. Philosophiques, 8(2), 259-272. https://doi.org/10.7202/203169ar

\section{Résumé de l'article}

Habituellement, les hommes politiques justifient ce qu'ils font en se référant à des idéaux reconnus. Il arrive que les idéaux invoqués correspondent à des intentions ou à des objectifs réels. Mais les invoquer, c'est aussi un moyen de propagande, un moyen de gagner du pouvoir. Dans cet article, il est question des ambiguïtés et des limites du discours éthique dans la pratique politique. Il s'agit non seulement de comprendre que ce discours peut être plus ou moins rusé ou mystificateur. Il s'agit aussi de dénoncer des idéaux unilatéraux ou démesurés. Il en est de grandiloquents, qui mobilisent et enthousiasment jusqu'au fanatisme des partisans, qui mettent en danger la liberté de tous et la paix de la cité. 


\title{
LE POUVOIR DE L'IDÉAL ET L'IDÉAL DU POUVOIR
}

\author{
par Joseph Pestieau
}

\begin{abstract}
RÉSUMÉ. Habituellement, les hommes politiques justifient ce qu'ils font en se référant à des idéaux reconnus. Il arrive que les idéaux invoqués correspondent à des intentions ou à des objectifs réels. Mais les invoquer, c'est aussi un moyen de propagande, un moyen de gagner du pouvoir. Dans cet article, il est question des ambiguïté et des limites du discours éthique dans la pratique politique. Il s'agit non seulement de comprendre que ce discours peut être plus ou moins rusé ou mystificateur. Il s'agit aussi de dénoncer des idéaux unilatéraux ou démesurés. Il en est de grandiloquents, qui mobilisent et enthousiasment jusqu'au fanatisme des partisans, qui mettent en danger la liberté de tous et la paix de la cité.
\end{abstract}

ABSTRACT. Political figures usually justify their actions by referring to established ideals. Sometimes these ideals do coincide with actual aims and intentions. But reference to such ideals is also a form of propaganda, a way of gaining power. This article discusses the limitations and ambiguities of ethical reference in politics. It goes beyond the demonstration that such reference can be guileful or manipulating to denounce ideals which are unilateral or immoderate. Some of these are grandiloquent and they mobilize and enthuse their adherents to the point of fanaticism thus endangering the liberty of all and the peace of the city.

Habituellement, les hommes politiques justifient ce qu'ils font en se référant à des idéaux reconnus. Il arrive que les idéaux invoqués correspondent à des intentions ou à des objectifs réels. Mais les invoquer, c'est aussi un moyen de propagande, un moyen de gagner du pouvoir. Dans ces pages, il sera question des ambiguïtés et des limites du discours éthique en politique. Il s'agira non seulement de comprendre que ce discours peut être plus ou moins rusé ou mystificateur. Il s'agira aussi de dénoncer des idéaux unilatéraux ou démesurés. Il en est de grandiloquents, 
qui mobilisent et enthousiasment jusqu'au fanatisme des partisans, qui mettent en danger la liberté de tous et la paix de la cité.

Traçons d'abord les perspectives et le plan de l'argument. Groupes de pression, partis et gouvernements, tous prétendent que leur cause est juste et le croient sans doute. En reprenant et en manipulant des idées reçues, ils donnent et se donnent de bonnes raisons de lutter dans tel ou tel sens. Ils présentent leurs besoins et leurs intérêts dans la perspective de valeurs avec lesquelles personne, à première vue, ne voudrait être en désaccord. Ces valeurs peuvent donner une noble signification aux besoins ou aux intérêts, les modeler et les transfigurer. Elles peuvent aussi être utilisées dans un discours mensonger afin de masquer ce qu'on poursuit effectivement et qu'on n'oserait avouer ou s'avouer. Mais avant de voir dans toute référence à des valeurs morales un alibi ou un artifice de la propagande, il faut reconnaître qu'elles ont une importance certaine puisque tant de gens se soucient d'être, au moins en apparence, en accord avec elles. C'est encore au nom de ces valeurs que des propagandes rivales dénoncent, chacune de son côté, les demi-vérités des autres. La référence de la politique à l'éthique n'est pas que faux-semblant.

Dans une démocratie, le public peut apprécier les positions des différents partis dans la mesure où les uns disent ce que les autres voudraient taire. Cependant, l'information du public, la pertinence et la pluralité des points de vue à partir desquels le public juge des positions des différents partis, dépendent de la propagande même de ces partis. La concurrence qu'ils se livrent, les programmes qu'ils défendent et les insuffisances dont ils s'accusent les uns les autres, sont une des sources principales des diverses opinions publiques. Les partis, dans leurs pratiques et leurs discours, visent à influencer le jugement de l'électorat et non seulement à s'y adapter.

Les idéaux qui ont cours en politique obéissent aux mêmes clivages et aux mêmes inerties que les partis. Ils sont un des principaux moyens d'identification des partis. En leur nom, on rassemble des partisans et on discrédite des opposants. Ils sont une arme du pouvoir avant même d'orienter l'action du pouvoir. Cela n'implique pas nécessairement qu'on trompe le public et qu'on ne fera pas ce que la propagande annonce. Pour pouvoir 
réaliser les idéaux dont parle la propagande, il faut bien que celle-ci ait d'abord réussi à rassembler des troupes autour des ces idéaux.

Les idéaux politiques commencent donc par servir le pouvoir qui est censé les servir. La plus noble cause requiert un pouvoir qui soit en mesure de la poursuivre. D'ailleurs, on s'associe d'autant plus volontiers à une cause qu'elle devient celle d'une force politique qui s'impose et réussira peut-être à s'imposer comme pouvoir légitime. Des partisans l'embrassent parce qu'ils savent qu'elle a les moyens de se réaliser. On comprend dès lors que l'idéal puisse être davantage l'instrument que le but du pouvoir qu'il justifie, dont il assure la bonne conscience et le bon renom.

Nous parlerons d'abord de l'émergence et de la genèse des idéaux politiques dans leurs relations aux besoins sociaux. Ensuite, nous aborderons la question du mensonge, des scléroses et de l'irréalisme éventuel de ces idéaux. Enfin, nous envisagerons les rapports difficiles entre fins et moyens et les dangers de l'idéalisme en politique.

Puisqu'il faut tenir compte des pressions les plus fortes pour maintenir un équilibre social et sauvegarder l'ordre, pourquoi ne pas assurer l'un et l'autre en satisfaisant tout le monde? La concorde qui s'ajouterait à la paix et la garantirait ne serait-elle pas un immense avantage pour tous? Les privilégiés ne renonceraient-ils pas volontiers à leurs privilèges s'ils gagnaient en fraternité ce qu'ils perdraient en pouvoir ou en richesse, s'ils étaient accoutumés à valoriser l'égalité plutôt que la puissance, l'entraide plutôt que l'accumulation privée, si, par-dessus le marché, ils n'avaient plus à se soucier de défendre des privilèges? Une solidarité réussie ne supprimerait-elle pas l'insécurité et ses séquelles, le besoin de dominer et d'accumuler aussi bien que le besoin d'être dominé ou encadré, que ce soit par des hommes ou des institutions? Des idéaux aussi immenses que ceux que nous venons de formuler semblent parfois opérer dans la pratique. Ils rallient des partisans à l'occasion de manques particuliers auxquels ils répondent en offrant une perspective d'espérance et d'action exaltante d'un point de vue éthique. C'est dans une telle 
perspective que des besoins et des ressentiments s'accusent ou qu'on ose les avouer. Ils provoquent en retour une première détermination de l'idéal.

C'est la rareté et la cherté du pain qui provoquent l'émeute, mais c'est au nom de la liberté, de l'égalité et de la fraternité que cette rareté et cette cherté ne sont plus tolérées, que les émeutiers se rassemblent et se font des alliés. Désormais, tout ce monde réclame bien plus et bien mieux que du pain à bon compte. Pourtant, c'est encore la faim et le ressentiment des affamés qui entretiennent l'effervescence idéaliste et obligent à persévérer dans le vouloir de l'idéal. En tout cas, il suffit parfois de repaître de pain les affamés de pain et de justice pour qu'ils n'aient plus faim ni de pain ni de justice.

L'idéal devient cause politique et mobilisatrice parce qu'il donne forme à des aspirations diffuses mais partagées, tout en répondant de façon qui semble adéquate à des besoins collectifs, qui deviennent d'autant plus pressants que l'on a l'espoir de les combler, que l'on voit comment les combler. L'idéal transforme la nature des besoins ressentis en les nommant, en montrant comment ils correspondent à des aspirations nobles, reconnues comme telles par la culture ambiante. On peut dire aussi qu'en donnant une forme publique à ces aspirations, l'idéal politique les fabrique de toutes pièces dans bien des coeurs. Que l'on songe à tous les gens en mal d'un parti ou de convictions, tout heureux de reprendre à leur compte des slogans et de nobles émois largement partagés qui ne contredisent pas leurs intérêts. L'expérience humaine y perd en authenticité et en variété, dira-t-on, mais des visées communes prennent ainsi la place de frustrations privées: des êtres isolés, sans voix et sans force, constituent désormais une voix et une force.

Un idéal correspond d'abord à une intention. Il lui faut ensuite se déterminer à travers des objectifs précis pour durer comme cause politique. Sa capacité mobilisatrice dépendra alors du réalisme, au moins apparent, de sa mise en oeuvre. C'est en dessinant un programme d'action qu'on mesure mieux les possibilités concrètes qui s'offrent. Si la pratique apporte mesure et pondération à l'idéal, elle lui permet aussi de se redéfinir, avec plus d'assurance et de précision, comme guide de la pratique. 
Il convient de bien préciser, les uns par rapport aux autres, les termes suivants: aspiration, besoin, intérêt, idéal (ou cause politique), objectif et programme de gouvernement. L'aspiration, voeu moral encore indéfini, peut cependant offrir un écho à la proclamation d'un idéal. Elle justifie alors l'adhésion à celui-ci et se précise en lui. L'idéal, pour opérer politiquement, doit exprimer des aspirations latentes, mais aussi répondre à des besoins bien concrets. Ceux-ci, et le cortège d'antagonismes et de ressentiments qui en découlent, sont les moteurs de toute action politique. Ils font la force des idéaux, mais ceux-ci, en répondant aux besoins, leur conferent une noble signification, une publicité et une nouvelle actualité. La faim se transfigure en faim de justice. Elle devient une exigence reconnue, prioritaire, mobilisatrice. Les besoins, évidemment, ne comptent que s'ils sont partagés et correspondent à l'intérêt d'un groupe qui sait se faire entendre. Besoins et aspirations s'objectivent publiquement dans des idéaux et des intérêts partagés, idéaux et intérêts qui se mêlent en un discours édifiant, qui donnent lieu aussi à des objectifs précis. Pour illustrer de façon cavalière ces distinctions, nous dirions que les prêtres entretiennent les aspirations, les prophètes et utopistes politiques nomment l'idéal et révèlent ainsi les besoins et les intérêts autour desquels des forces nouvelles pourront se regrouper, les chefs de parti proposent des objectifs et organisent des troupes. Si ces chefs deviennent les gouvernants, il leur faudra mettre au point des programmes législatifs et administratifs dans le prolongement de leurs programmes électoraux. Évidemment, une dialectique permanente s'établit entre ces diverses phases de ce qu'on pourrait appeler la détermination progressive d'un objectif politique.

Souvent un parti ou un gouvernement se contente de proclamer un objectif pour des raisons de propagande. Mais une fois qu'on l'a proclamé, on peut être obligé de l'instituer, car des attentes populaires que l'on a reconnues, auxquelles on a promis de répondre, deviennent des exigences qu'il sera de plus en plus difficile d'éluder.

Qu'un idéal soit le point de ralliement d'une opposition ou d'un gouvernement, il est dans les deux cas un moyen de gagner du pouvoir, pouvoir qui ne servira pas nécessairement à réaliser l'idéal. On promet des lendemains qui chantent à tous ceux qui 
sont insatisfaits du temps présent, mais à qui sert cette promesse? L'idéal au nom duquel on cherche à renverser un gouvernement n'est pas a priori plus véridique que celui qui sert de justification au pouvoir établi. Il est plus facile de faire des promesses en l'air quand on n'a pas encore les moyens de les tenir, quand on est dans l'opposition plutôt qu'au gouvernement. Par ailleurs, une des armes de l'opposition est de montrer l'écart entre les idéaux que proclame le gouvernement et la politique qu'il poursuit. C'est ainsi qu'elle peut acculer le gouvernement à faire ce qu'il dit ou le défaire.

Parlons des mirages de l'idéal. Ses promesses peuvent être un guide pour l'action. Elles sont aussi une consolation dans les tribulations présentes. On les célèbre, et cela est bien nécessaire pour tremper la résolution et la solidarité des partisans. Mais ne risque-t-on pas d'en oublier les exigences quotidiennes de la lutte et de l'organisation politiques? Il est tentant de se complaire dans le rêve d'une utopie et de fulminer contre le monde présent. Il est plus difficile de se réconcilier avec celui-ci et de tâcher d'y faire advenir patiemment l'utopie dont on rêve. Pour inspirer des stratégies, un idéal doit se nourrir des possibilités du moment, se redéfinir sans cesse pour exploiter ces possibilités. Or, les mouvements de masse, plus encore que les individus, ont besoin de dogmes. Plus exactement, les individus comptent sur les mouvements dont ils font partie pour y trouver à la fois sécurité affective et certitudes morales. Ce que les partisans veulent de leur parti, surtout dans le cas de partis extrémistes, n'est-ce pas, au moins pour une part, le réconfort d'une vision du monde arrêtée, d'une espérance assurée et d'un sentiment de justification (garanti par l'adhésion à la discipline du parti)? L'idéal met en perspective un manque particulier, donne du souffle et de l'audace à la revendication. Il reprend la particularité de celle-ci dans une visée générale mais peut aussi la noyer dans des promesses grandiloquentes. Pour prendre un exemple extrême, ceux qui s'absorbent dans l'espérance messianique d'un renversement justicier ne sont guère préparés à exploiter les chances limitées qui s'offrent au jour le jour pour réaliser des réformes modestes. Cela leur paraîtrait une trahison du rêve auquel ils se sont identifiés.

Les mirages de l'idéal peuvent être entretenus délibérément ou exploités pour entretenir le pouvoir des uns sur les autres. C'est 
en proclamant des objectifs aussi séduisants que vagues, qu'une politique habile attire et manipule des supporters dont les intérêts sont pourtant étrangers à ceux que poursuit activement cette politique. Ainsi, la bourgeoisie a neutralisé ou même utilisé des mouvements ouvriers formés contre elle en invoquant la nécessité de redresser la productivité et la prospérité nationales, alors que les bénéfices de celles-ci étaient d'abord réservés à la bourgeoisie. Il arrive aussi que des appareils revendicatifs en place utilisent une rhétorique révolutionnaire maximaliste afin de faire dériver, selon leur convenance, les revendications précises de la base. Il y a des partis révolutionnaires institutionnalisés et des syndicats bureaucratiques, au pouvoir comme dans l'opposition, qui monopolisent le discours contestataire de telle sorte qu'ils créent l'illusion de contester effectivement et évitent ainsi d'assumer les contestations qu'ils ne veulent pas entendre. Ils discréditent ou suppriment les dissidents et continuent d'occuper tout l'horizon de la gauche avec leurs mots et leurs mensonges.

Il est utile de distinguer trois phénomènes, quoiqu'ils se recoupent et s'épaulent très souvent:

a) L'idéologie habile et fallacieuse par laquelle une classe (groupe ou nation) arrive à manipuler à son avantage une autre classe (groupe ou nation) en manipulant l'interprétation que celle-ci a des faits et des valeurs.

b) L'interprétation des faits et des valeurs, traditionnelle, sclérosée et inadéquate, dont on ne se départit pas parce qu'on y trouve le réconfort de certitudes habituelles.

c) L'idéalisme éthéré, incapable de se traduire en politiques concrètes, qui assure cependant un refuge contre la morosité et les complexités du présent.

On réserve habituellement le mot idéologie pour désigner le phénomène mentionné en a). Dans ce cas, l'aveuglement est entretenu, mais pas nécessairement consciemment, afin de maintenir un rapport de force. Il fait partie d'un système de domination ou d'exploitation ${ }^{1}$.

1. L'idéologie comme mystification au service d'un système de domination, composante et légitimation d'un tel système, n'est dénoncée que par une autre idéologie, établie ou tâchant de s'établir. La dénonciation est toujours relative à un point de vue. Cela ne la discrédite d'ailleurs pas automatiquement. D'autre part, une idéologie, avant d'être mystificatrice, est la vision du monde qui permet à un milieu de s'identifier et de défendre ses intérêts. Tout point de vue est idéologique, partiel et partial. Le philosophe peut le savoir, il n'y échappe pas. Sa critique des idéologies est encore inhérente à une idéologie. 
On peut critiquer un même phénomène de ces trois points de vue, ou de deux de ces trois points de vue. Une religion, par exemple, peut être, en même temps, idéologie conservatrice utilisée plus ou moins sciemment pour embobiner les désavantagés; espérance sans bavure d'un renversement justicier que les désavantagés attendent, mais sans le préparer efficacement; une vision du monde arrêtée, rassurante, établie dans des dogmes, des rites et des institutions, incapable de rendre compte des dynamismes et des possibilités politiques actuels. Elle est une excellente idéologie parce qu'elle est aussi vision rassurante ou espérance passive et magnifique. Cette même religion peut être pour certains, ou avoir été à une autre époque, une source d'idéaux agissant à l'avant-garde de l'histoire. C'est d'ailleurs cet aspect «progressiste» de la religion qui lui donne la capacité d'abuser les désavantagés et rassure la conscience des fidèles qui ne voudraient pas ne pas être "progressistes». Bref, pour qu'une idéologie puisse s'accréditer et tromper, il faut bien qu'elle ait le prestige de la tradition établie ou l'apparence de la grandeur morale ou l'un et l'autre. D'autre part, une vision du monde qui se maintient envers et contre les intérêts des uns, est sans doute entretenue et cultivée par d'autres qui y trouvent avantage. Elle dure d'autant plus qu'elle est reprise dans une perspective idéologique.

La propagande par laquelle un parti tâche de gagner de nouveaux appuis est très souvent indistinctement le discours par lequel le même parti se retranche dans l'assurance de sa bonne $\mathrm{foi}^{2}$. La propagande est à la fois tournée vers l'intérieur et l'extérieur. On veut convaincre les autres ou les abuser, mais on a aussi besoin de s'abuser soi-même, de se convaincre que l'on est en accord avec ses propres principes. Des partisans ont besoin de convictions nettes, voire manichéennes, qui leur donnent le beau rôle. Et pour cela, ils négligent d'envisager tous les aspects et toutes les ambiguiités de leur position. Il devient parfois difficile de distinguer, comme nous le faisions plus haut, entre la ruse mensongère de la propagande, l'identification "sincère» à des idéaux inopérants devenus habituels et l'évasion dans un rêve consolateur.

2. Bonne foi entretenue éventuellement avec plus ou moins de mauvaise foi par des cadres et des idéologues. 
Concluons. Ceux qui ne réclament que du pain ici et maintenant risquent fort de demeurer isolés et donc faibles dans leur revendication particulière. Ils ne réclament même pas l'abolition de leur statut de quémandeurs. Par contre, ceux qui placent leur revendication dans la perspective d'un idéal général, risquent de s'égarer dans les mots. Pour opérer dans la pratique comme anticipation directrice, il faut qu'un idéal non seulement enthousiasme et rassemble des fidèles, mais aussi se concrétise dans une stratégie répondant à des besoins ressentis. En retour, cette stratégie, par son efficacité à résoudre des maux réels, ajoutera à la force d'entraînement de l'idéal qui l'inspire. D'autre part, en se situant dans la perspective d'idéaux reconnus, la défense d'intérêts particuliers gagne de la respectabilité et de nouveaux soutiens. Une politique ne s'impose pas seulement par la force mais aussi par la justification qu'elle donne d'elle-même et par les fins qu'elle affiche. Plus exactement, une politique peut tirer de cette justification et de ces fins, la force par laquelle elle s'imposera. On voit quels sont le rôle, la nécessité et les tentations de la propagande. Celle-ci, sans être nécessairement mensonge délibéré, ne dit ni toute la vérité ni rien que la vérité. Pourtant, c'est dans la concurrence des différentes propagandes que leurs fauxsemblants, leurs partis-pris et leurs excès sont dénoncés de façon fort pratique.

Nous venons d'insister sur les rôles souvent ambigus de l'idéal dans les discours politiques. Nous allons maintenant parler brièvement des moyens spécifiques de la politique: la contrainte de la loi et une certaine duplicité ou ruse, moyens que nécessite le maintien de la paix, moyens qui peuvent entrer en contradiction avec certains idéaux.

Un gouvernement doit bien agir par la contrainte. Celle-ci est nécessaire parce que la loi, fort souvent, n'a d'efficacité qu'imposée dans sa totalité et à tous. Ainsi, le code de la route doit s'appliquer intégralement et universellement parce qu'il constitue un ensemble de règlements interdépendants, ne garantissant la sécurité de la route que si tous les conducteurs s'y conforment. On ne peut vraiment pas compter sur le bon vouloir, toujours aléatoire, de tous les conducteurs, à tout moment, vis-à-vis de tous les règlements. D'autre part, c'est parce que le citoyen 
ordinaire est convaincu que tout le monde observe la loi, qu'il est disposé à en faire autant. Ainsi, chacun garde sa confiance dans l'ordre légal, estime qu'il vaut la peine d'y contribuer et craint d'être l'unique déviant, cible de la police ou de la réprobation générale, au cas où il ne s'y conformerait pas. Il est donc important d'imposer la loi sans faille, manu militari au besoin, afin qu'elle forme un système efficace de règlements, un système majestueux qui s'impose sans conteste. Les contrôles policiers sont toujours onéreux et souvent vexatoires, leur multiplication n'ajoute pas à la majesté de la loi. Pourtant, cette majesté repose sur une application rigoureuse et universelle de la loi, sur une crainte diffuse de l'enfreindre. Évidemment, l'imposition par la force d'un cadre légal peut assurer l'ordre et la paix, mais non la fraternité, même si celle-ci est l'objectif visé par la loi. La contrainte est un moyen qui ne se prête pas à toutes les fins. On peut en dire autant de bien d'autres moyens auxquels doivent recourir les hommes politiques les mieux intentionnés.

Poursuivre un idéal en politique, c'est aussi poursuivre le pouvoir ou au moins l'influence qui permettra de mettre en oeuvre cet idéal. Voilà qui exige que l'on négocie, que l'on transige avec des adversaires, que l'on ruse pour se ménager des appuis, que l'on suscite des espérances qui ne sont pas toujours mesurées, que l'on manipule des partisans, que l'on sabote l'opposition, que l'on ne perde ni du temps ni des occasions à cause de scrupules. Il faut être roublard, impitoyable, opportuniste pour les meilleures causes comme pour les moins bonnes. Machiavel a fort bien dit ces choses ${ }^{3}$. Le métier de politique comporte des exigences telles que, très souvent, on se laisse absorber par le souci de se maintenir au pouvoir, d'imposer un minimum d'ordre, d'expédier les affaires courantes. On n'a guère le loisir de viser plus loin et plus haut quoique, pour des raisons de propagande, ne fût-ce que pour rester en selle, il faille bien parler et parfois s'occuper du long terme et d'idéaux élevés.

Par contre, si l'on vise trop loin et trop haut avec entêtement, si l'on veut transformer coûte que coûte le corps social et faire fi de ses habitudes, les moyens utilisés risquent de contredire les objectifs recherchés. On a recouru à la terreur pour imposer la fraternité, à l'épargne et au travail forcés pour assurer la libération économique du peuple, à la guerre pour préparer la paix. Mais on

3. Cf. Le Prince, chap. XV à XIX. 
s'est installé dans la terreur et la fraternité fut à jamais corrompue. Dans la guerre et la paix fut oubliée. Dans l'épargne et le travail forcés et la libération du peuple ne fut plus qu'une mauvaise farce, un alibi pour perpétuer l'oppression à laquelle des oppresseurs se sont habitués. Les plus idéalistes, dans l'impatience de réaliser leurs objectifs, deviennent souvent cruels et totalitaires de façon irrémédiable. Au début, la grandeur de leurs desseins semble justifier leur intransigeance. Et puis il y a toujours, à tous les échelons de l'appareil de l'État, des hommes qui trouvent dans l'exercice du pouvoir et dans la démesure de celui-ci, des avantages qu'ils s'emploieront à maintenir. Le despotisme s'établit progressivement. Il entraîne tant de crainte et de ressentiment que les despotes n'osent plus sortir de ce mode de gouvernement même s'ils souhaitent restaurer la fraternité et la liberté. Le peuple qui ne participe pas aux décisions politiques ne se prépare pas aux vertus républicaines et les despotes, dès lors, ont quelques raisons de le traiter en mineur.

En dépit de la contrainte des lois, des ruses du pouvoir et des luttes plus ou moins sourdes pour le pouvoir, des passions soulevées et des espérances trompées, bref, en dépit de ses moyens qui semblent amoraux ou immoraux, la politique demeure pourtant passible d'un jugement éthique. En quel sens et dans quelle mesure? C'est ce que nous allons préciser.

Normalement, un gouvernement parle de ses bonnes intentions. Il lui est plus économique de se faire respecter pour ses nobles desseins, réels ou prétendus, que de se faire craindre par une police, de justifier l'ordre imposé que de l'imposer par la seule force. D'ailleurs, il ne suffit pas de bien payer la police, il faut encore donner un sens à sa mission pour que ses agents gardent l'estime d'eux-mêmes et le coeur à l'ouvrage. La propagande et la contre-propagande, qu'elles soient mensongères ou sincères, en appellent aux valeurs éthiques parce que celles-ci représentent une dernière instance. En fait, ces valeurs, pour le philosophe comme pour n'importe quel citoyen, n'apparaissent pas indépendamment de l'influence des idéologies, des partialités ou des simplismes de la propagande. Il n'y a pas une vision des enjeux et des objectifs de la politique, qui s'imposerait en dehors des différentes opinions partisanes qui se font concurrence dans le public et essaient de se faire valoir, s'appuyant sur les institutions 
et les pouvoirs en place, mêlant la menace et l'argument, la bonne et la mauvaise foi. Ce n'est qu'en dénonçant les mensonges et les ambiguïtés de ces opinions, en les confrontant les unes aux autres, qu'on acquiert des idées plus justes au sujet des valeurs, des objectifs et des enjeux de la politique. Il faut bien reconnaître que le point de vue des opprimés, si juste soit-il, n'est remarqué que le jour où leur protestation fait les manchettes des journaux, parce que cette protestation sert la stratégie d'un parti établi ou parce qu'elle s'est exprimée de façon retentissante. Le moraliste ne s'est intéressé aux Noirs, aux Chinois, aux ouvriers, aux prisonniers de droit commun qu'après qu'ils se soient mis eux-mêmes à la mode par leurs révoltes ou leurs révolutions. Cela signifie que le pluralisme et la concurrence des points de vue, qui sont la condition de la critique des idéologies, reposent sur une répartition minimale des moyens de lutte.

Nous venons d'insister sur la difficulté d'un jugement équilibré en politique, mais aussi sur la référence permanente à l'éthique qu'on y rencontre. En fin de compte, une certaine législation promeut uen certaine conception de la société et de l'homme, ouvre certaines possibilités et en ferme d'autres, favorise des valeurs et en défavorise d'autres. Selon Julien Freund ${ }^{4}$, la politique inscrit ses divers objectifs dans la perspective de la paix, et celle-ci, qui est le but spécifique de la politique, se justifie dans la perspective des fins publique et privée qu'elle autorise, dans la perspective de la justice et de la liberté qui peuvent s'épanouir pour tous et pour chacun dans la paix. Si l'on distingue le but spécifique, la paix, et les fins qui la justifient, vis-à-vis desquelles la paix n'est qu'un moyen ou une condition, c'est qu'on veut dire deux choses. Premièrement, que la paix idéale n'est pas n'importe laquelle mais une paix qui favorise certaines fins. Deuxièmement, qu'il serait dangereux que le pouvoir politique cherche à imposer plus que la paix, plus qu'un ordre public où soit possible la coexistence d'opinions et de façons de vivre différentes. Il y a plusieurs raisons à cela. Une première série de raisons invoque les coûts excessifs d'une politique qui veut imposer plus que la paix. Une seconde série de raisons porte sur la nécessité d'un débat libre et ouvert au sujet des valeurs.

4. Cf. Chap. I, Qu'est-ce que la politique?, Le Seuil, Paris, 1967. 
D'abord, comment contrôler la vie privée et la conscience des citoyens? Si l'État s'y essaie, ce ne peut être qu'à un prix très élevé. En tout cas, on ne peut imposer par un règlement de police ni la liberté intérieure ni la droiture du coeur. L'État ne peut qu'assurer les conditions favorables à celles-ci. Il ne peut régler que l'extériorité des conduites à moins d'avoir l'arbitraire pour règle, la délation et le lavage de cerveau comme méthodes. Comme nous le disions plus haut, on ne peut imposer au corps social des idéaux dont il ne veut pas à moins de l'opprimer. Un gouvernement qui voudrait instaurer, par la force, ce qu'il entend par la vertu, risque de faire plus de mal que de bien et de se fourvoyer dans une voie unilatérale. La terreur et l'impatience de la vertu ont partie liée. Bien sûr, la paix la plus permissive ne va pas sans règles ni sans contraintes, mais il importe de mesurer celles-ci par rapport à la liberté qu'elles autorisent. On dit qu'Élisabeth $I$, agacée par les querelles interminables des théologiens au sujet de l'emplacement de l'autel dans les églises, aurait décidé de cet emplacement non pour des raisons théologiques, mais pour éviter une guerre de religion en imposant sa volonté arbitraire et souveraine. Il y avait dans cette mesure royale, qui remettait les théologiens à leur place, une garantie de tolérance pour tous.

Milovan Djilas écrivait: «Je suis convaincu que la société ne peut pas être parfaite. Les hommes doivent tenir à leurs idées et à leurs idéaux, mais ils ne doivent pas s'imaginer que les uns ou les autres soient réalisables. Il nous faut comprendre la nature de l'utopie. L'utopiste une fois au pouvoir devient dogmatique et il peut très facilement faire le malheur des hommes au nom de son idéalisme ( . . .). Le devoir de l'homme de notre temps est d'accepter comme une réalité l'imperfection de la société, mais aussi de comprendre que l'humanisme, les rêves et les imaginations humanitaires sont nécessaires pour le réformer sans cesse, pour l'améliorer et le faire progresser ${ }^{5} »$. Malheureusement, toute utopie ou sujet de la société parfaite peut devenir tyrannique dès qu'elle a les pouvoirs de s'implanter. L'idéal en la matière est sans cesse à réinventer à partir des insuffisances que révèlent son actualisation même et les besoins des hommes. Que serait une justice dont chacun ne pourrait pas redéfinir les conditions? La République de Platon ne ressemblerait-elle pas au Meilleur des mondes ${ }^{6}$ une fois

5. Milovan DJILAS, La Société imparfaite, Calmann-Lévy, Paris, 1969, pp. 16-17.

6. Celui d'Aldous HUXIEY. 
réalisée? L'anticipation du philosophe deviendrait de l'entêtement imbécile si elle refusait la contestation des citoyens, de la tyrannie si le philosophe entêté était roi, une tyrannie d'autant plus insidieuse qu'elle se ferait passer pour vertueuse et jetterait le discrédit sur toute dissidence.

Qui juge la politique d'un point de vue éthique estime à juste titre en juger en dernier ressort. Mais il faut rappeler, et c'est là-dessus que je voudrais conclure cet article, combien le jugement éthique dépend de la reconnaissance tâtonnante des besoins et des droits, combien cette reconnaissance est elle-même liée aux rapports mouvants qu'entretiennent les différentes forces et les différents discours politiques. Il faut aussi rappeler combien ces discours correspondent à une volonté d'auto-justification et de propagande. Chaque parti veut se donner bonne conscience en même temps qu'il essaie de défendre sa vision des choses. Il s'enferme volontiers dans celle-ci parce qu'elle lui offre une assurance morale. Pour sauvegarder cette assurance, et non seulement pour défendre ses intérêts, il sera aveugle aux points de vue et aux intérêts des autres.

Les nécessités immédiates du gouvernement, la volonté de garder le pouvoir ou de maintenir la paix sociale, obnubilent souvent les gouvernants. Mais les plus beaux idéaux révolutionnaires obnubilent aussi leurs partisans. Ils justifient qu'on leur sacrifie tout. Ils permettent d'oublier que le droit ne peut être dit une fois pour toutes, que la société n'est pas une mais multiple ${ }^{7}$ dans ses revendications et ses aspirations les plus légitimes.

Département de Philosophie

Collège de Saint-Laurent

7. Voir à ce propos, l'article de C. Lefort “Droits de l'homme et politique» in Libre, 80-7. Paris, Payot 1980 . 